\title{
Hybridintegration von Mikrodehnungssensoren
}

Th. Frank ${ }^{1}$, A. Grün ${ }^{1}$, M. Kermann ${ }^{1}$, A. Cyriax ${ }^{1}$, A. Steinke ${ }^{1}$, Th. Ortlepp ${ }^{1}$, G. Reschke ${ }^{2}$

${ }^{1}$ Forschungsinstitut für Mikrosensorik, Konrad-Zuse-Straße 14, D-99099 Erfurt, Deutschland, tfrank@cismst.de

${ }^{2}$ Technische Universität Dresden, Fakultät Maschinenwesen, Institut für Fertigungstechnik, GeorgeBähr-Straße 3c, 01069 Dresden, Deutschland

\section{Zusammenfassung}

Silizium-Dehnungsmesssensoren oder Siliziumdehnmessstreifen (Si-DMS) substituieren in einige Anwendungen, insbesondere im Bereich Druckmesstechnik, die piezoresistiven Dünnschicht- und die Dickschichtwiderstände. Der höhere Koppelfaktor und die deutlich höhere Langzeitstabilität machen diese Sensoren zunehmend interessant für weitere Anwendungen. Besonders hohe Anforderungen an die Montage bereitet die zunehmende geometrische Größe der Prüfkörper. Die Prozesse, wie sie für kleine Prüfkörper üblich sind, z.B. Glasfritt- und Drahtbonden, stoßen schnell an ihre Grenzen. Thermische Prozesse sind aufgrund der Masse des Prüfkörpers unwirtschaftlich oder nicht gewünscht. Die Drahtbondprozesse an den großen Prüfkörpern sind sehr aufwendig und sollen vermieden werden. Die Prüfkörper sind lange Rohre, Druckbehälter, Maschinenschrauben oder Federkörper für Kraftsensoren. Für die Anwendung sollen die Sensoren direkt montiert und so hybrid integriert werden. Der Beitrag beschreibt verschiedene Strategien zum Aufbau makroskopischer Prüfkörper mit hybrid integrierten Siliziumdehnmessstreifen.

Keywords: Silizium, Dehnmessstreifen, mechanische Spannung, Zustandsüberwachung, piezoresistiv

\section{Einleitung}

Silizium-Dehnungsmesssensoren ersetzen insbesondere im Bereich der Druckmesstechnik, die piezoresistiven Dünnschicht- und die Dickschichtwiderstände. Durch die geringe geometrische Größe des Prüfkörpers sind die notwendigen Prozesse wie, Drahtbonden und thermische Fügeprozesse sehr einfach auszuführen sind. Somit sind sie unter anderem als Drucksensoren prädestiniert. Der höhere Koppelfaktor und die deutlich höhere Langzeitstabilität machen diese Sensoren zunehmend interessant für weitere Anwendungen. Besonders hohe Anforderungen an die Montage bereitet die zunehmende geometrische Größe der Prüfkörper. Die Prozesse, wie sie für kleine Prüfkörper üblich sind, z.B. Glasfritt- und Drahtbonden, stoßen schnell an ihre Grenzen. Thermische Prozesse sind aufgrund der Masse des Prüfkörpers unwirtschaftlich oder nicht gewünscht. Die Drahtbondprozesse an den großen Prüfkörpern sind sehr aufwendig und sollen vermieden werden, da sowohl die Temperaturbelastung nicht gewünscht ist, als auch die vollständige Erwärmung dieser Bauteile hohe Kosten verursacht. Die Prüfkörper sind oft lange Rohre, Druckbehälter, Maschinenschrauben oder Federkörper für Kraftsensoren. Im Folgenden werden verschiedene Strategien zum Aufbau makroskopischer Prüfkörper mit hybrid integrierten Siliziumdehnmessstreifen untersucht.

Bei der am häufigsten verwendeten Dopingvariationen, werden die resistiven Elemente im nTyp-Basismaterial durch Dotierung typischerweise mit Bor realisiert. In diesem Fall sind die piezoresistiven Koeffizienten, quer $\left(\pi_{T}\right)$ oder längs $\left(\pi_{\llcorner}\right)$in Bezug auf die Stromrichtung vom Betrag nahezu gleich, aber entgegengesetzt gerichtet. Für einen einachsigen Spannungszustand kann daher eine Messbrücke aufgebaut werden, indem zwei Widerstände parallel und zwei Widerstände senkrecht zur angelegten mechanischen Spannung oder Belastung angebracht sind. Dies ermöglicht ein hohes $\mathrm{MaB}$ an Miniaturisierung. Eine Fläche von 100 $\mu \mathrm{m}$ mal $100 \mu \mathrm{m}$ reicht für eine WheatstoneBrücke aus.

Mit Hilfe von MEMS-Technologien werden die Si-DMS hergestellt. In Abb. 1 ist das Layout und die Schaltung dargestellt. Die Implantationsparameter sind für einen Flächenwiderstand des piezoresistiven Widerstandes von ca. $200 \Omega$ mit einem Temperaturkoeffizienten von ca. 0,8\% / K ausgelegt. Die piezoresistiven Widerstände sind durch hoch implantierte Leitungen mit den Bondpads verbunden.

Die Chipfläche des Si-DMS beträgt 500 x 500 $\mu \mathrm{m}^{2}$. Für die verschiedenen Anwendungen können die Sensoren bis zu $5 \mu \mathrm{m}$ abgedünnt werden. Diese Variante ist sehr gut geeignet, 
die mechanischen Spannungen in einem homogenen Spanungsfeld zu bestimmen.

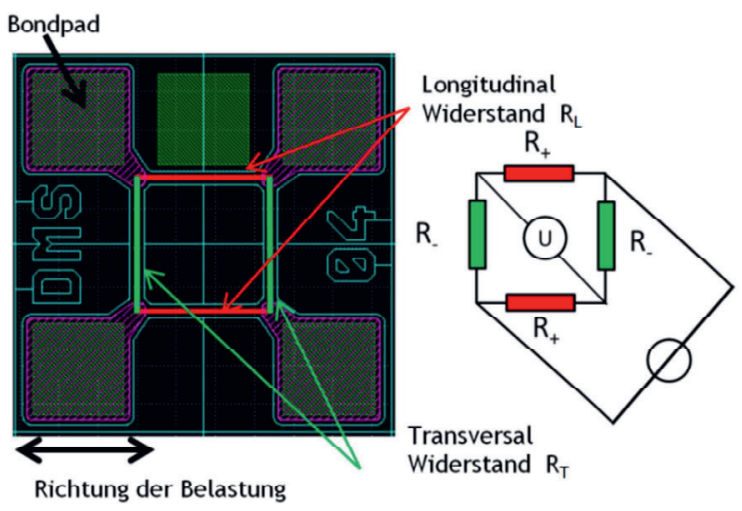

Abb. 1: Si-DMS mit kompletter Messbrücke mit zwei Widerständen für den Längs- und den Quereffekt

Durch die Anordnung der Messwiderstände ist der Si-DMS in der Ebene in alle Richtungen gleich empfindlich. Die durch unterschiedlichen Temperaturausdehnungskoeffizienten induzierten mechanischen Spannungen können so leicht kompensiert werden. Für die Positionierung des Sensors ist aber eine gute Kenntnis der mechanischen Belastung notwendig. Eine optimale Position weißt eine möglichst hohe Anisotropie der mechanischen Spannungen auf.

\section{Montage Si-DMS aus Stahl}

Die Si-DMS werden auf verschiedenen Federkörpern mittels Glasfritte montiert, die Verarbeitungstemperatur beträgt ca. $450{ }^{\circ} \mathrm{C}$.

Aufgrund der hohen Unterschiede in der thermischen Ausdehnung zwischen metallischen Federkörpern und dem Silizium steht der DMS unter starken lateralen Druckspannungen. Diese beeinflussen natürlich auch die Messwiderstände, die zu einer Messbrücke verbunden sind. Die Messbrücke kompensiert die Belastung nahezu vollständig, wenn die Bedingungen an allen Messwiderständen identisch sind. Dies wird durch ein völlig symmetrisches Design erreicht.

An die Verbindung werden sehr hohe Anforderungen gestellt. Diese muss insbesondere langfristig stabil sein. Dies bedeutet, dass die mechanische Belastung vom Federkörper auf den Si-DMS ohne Dehnungsverluste übertragen wird. Temperaturschwankungen dürfen keine Hysterese verursachen. Das Kriechen aufgrund der hohen mechanischen Belastung ist auszuschließen. Als Werkstoff für den Federkörper eignen sich Aluminium, Titan und Stahl. Eine sichere Verbindung, welche die Anforderungen erfüllt, ist eine Verbindung mittels Glasfritte [1], [2].

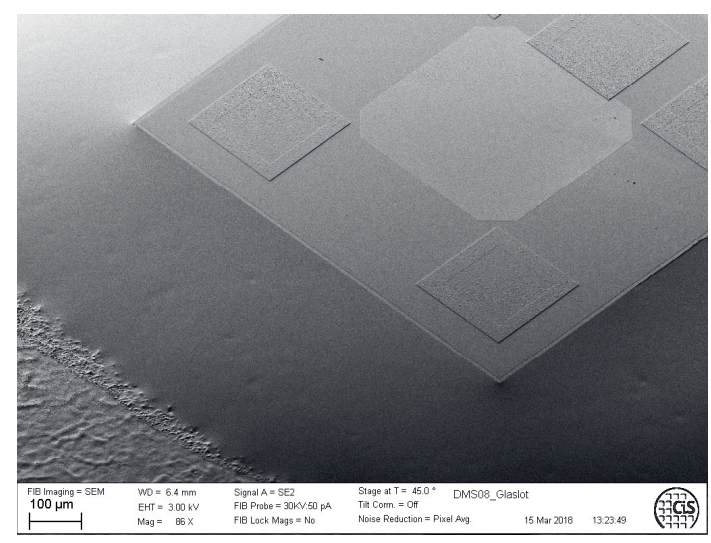

Abb. 2: Auf Stahl (1.4542) mit Glasfritte gefügter Si-DMS

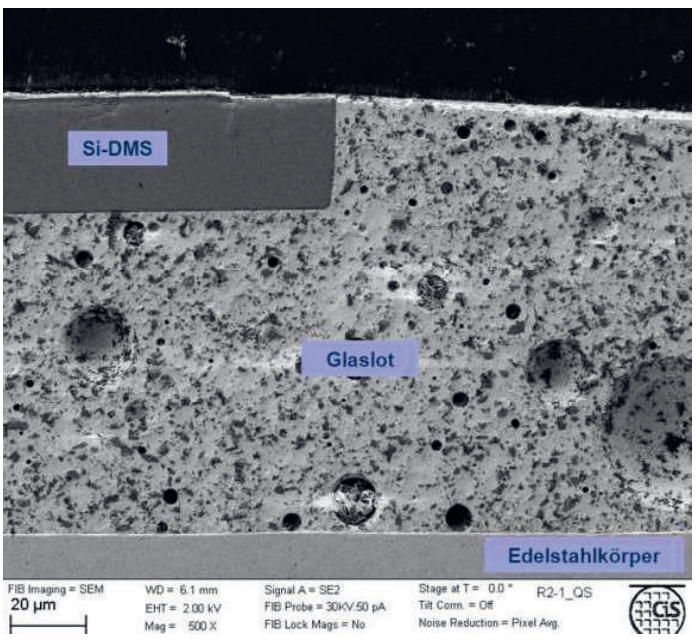

Abb. 3: Schnitt durch die Verbindung

Abb. 2 und Abb. 3 zeigen eine REM Aufnahme einer Glaslotverbindung mit einem bleihaltigen Glaslot. In [3] wurde bereits das Ergebnis einer Langzeituntersuchung gezeigt, nach $24 \mathrm{~h}$ betrug die Abweichung weniger als, 0,01\% der Messspanne.

\section{Simulation der Belastung}

Als Beispiel dient eine Schraube als Prüfkörper bzw. Federelement.

\section{Die Schraube als Verbindungselement}

Sollen die Belastung eines komplexen Bauelementes, welche durch äußere und innere Kräfte wirken, gemessen und auch aufgezeichnet werden, eignen sich hierfür besonders die Verbindungselemente, da sich die Belastungen an diesen konzentriert. Als Beispiel sollen die Maschinenschrauben dienen.

Eine Schraube ist ein Maschinenelement zur kraftschlüssigen Verbindung von zwei oder mehr Bauteilen. Durch das Anzugsmoment wird zwischen Schraubenkopf und Muttergewinde die Montagevorspannkraft aufgebaut. 
Die maximale zulässige Vorspannkraft wird durch den Bolzendurchmesser und die Festigkeitsklasse der Schraube definiert. Für eine dauerhaft sichere und funktionsfähige Verbindung ist zu gewährleisten, dass die Vorspannkraft über die gesamte Lebensdauer in einem definierten Toleranzbereich liegt.

Für Standardanwendungen mit bekannten Belastungen, ist die Einhaltung des Anzugsmomentes und der Richtlinie VDI 2230 ausreichend. Besonders sicherheitsrelevante Verbindungen bedürfen jedoch regelmäßiger Kontrollen. Die nachträgliche Kontrolle der Montagevorspannkraft ist ohne verschiedene $\mathrm{Zu}$ satzmaßahmen nicht möglich. Standardgemäß wird das Anzugsmoment manuell überprüft. Es ist zudem auszuschließen, dass Korrosion und Verschmutzung durch Erhöhung der Reibung ein korrektes Anzugsmoment vortäuschen. Vorteilhaft ist das Monitoring der Montagevorspannkraft. Die Vorspannkraft dehnt den Schraubenschaft, staucht die zu fügenden Elemente und deformiert sowohl den Schraubenkopf als auch das Muttergewindes. In der Deformation des Schraubenkopfes, bzw. der Mutter sind damit alle Informationen über die Vorspannkraft enthalten. Für die Messung haben sich Dehnmessstreifen als vorteilhaft erwiesen. Sie bilden die Deformation präzise ab.

\section{Belastung der Schraube}

Zwischen dem Schraubenkopf und dem Muttergewinde wird durch das Anzugsmoment die Montagevorspannkraft aufgebaut. Besonders an der Oberfläche des Schraubenkopfes ist die resultierende mechanische Dehnung, bzw. mechanische Spannung für die Si-DMS leicht zugänglich. In Abb. 4 ist die Belastung des Schraubenkopfes dargestellt. Insbesondere die mechanischen Spannungen diagonal über den Kopf sind von Interesse.

Die Abb. 5 zeigt die mechanischen Spannungen in Richtung x (blau) und y (grün). Die optimale Position liegt bei der betragsmäßig größten Differenz beider Spannungen, die lokalen Minima der roten Linie, hier ist die Anisotropie am größten. Dieser Bereich liegt ungefähr an der Stelle wo der Schraubenbolzen den Kopf durchdringt. Dies würde bedeuten, dass sich für jede Schraubenabmessung die Position ändert. Wünschenswert ist eine Montage des Sensors in der Mitte der Schraube. Dazu ist es erforderlich die auftretenden mechanischen Spannungen in einer Richtung vom Sensor fernzuhalten. Im Beispiel sind dies die Spannungen in Richtung y.

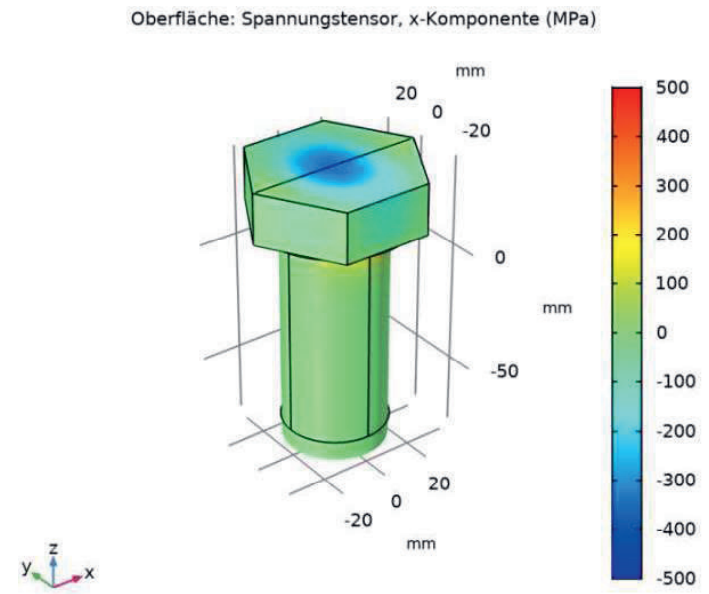

Abb. 4: FEM-Simulation Schraubenkopf

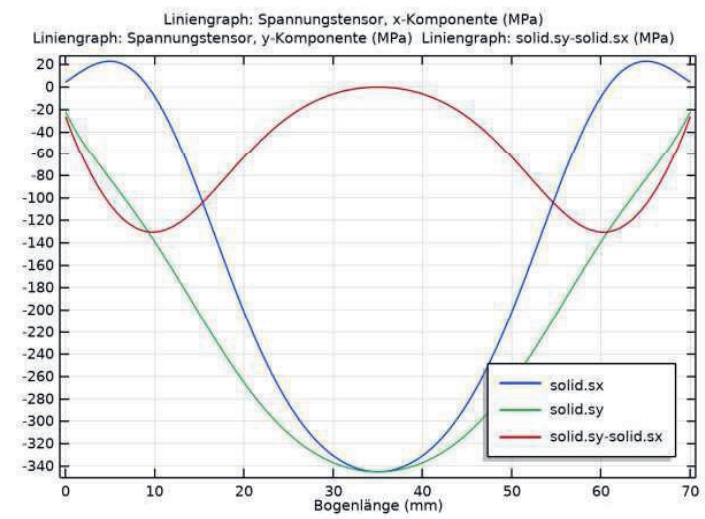

Abb. 5: Simulierte mechanische Spannung über dem Schraubenkopf, M42 nach DIN 931

\section{Die Kompensation der Querspannung}

Die Kompensation der Querspannung kann leicht erreicht werden, wenn der gefügte SiDMS auf dem Federkörper links und rechts in Richtung y durch eine Nut vom Kraftfluss getrennt wird. Die Nut kann direkt in den Federköper eingearbeitet oder über einen Montageträger gefügt werden. Die Abb. 6 und Abb. 7 zeigen die Verteilung der mechanischen Spannung. Gut zu erkennen ist die Filterwirkung des Stegs, bzw. der Nuten.

Abb. 8 und Abb. 9 zeigen in einem Diagramm den Verlauf beider Spannungen. Der Unterschied besteht darin, dass in Abb. 8 ein Montageträger verwendet wurde, hingegen sind in Abb. 9 die Nuten direkt in den Schraubenkopf geschnitten. Die Funktion des Montageträges ist in [3] und [4] bereits erläutert. Durch die Verwendung des Montageträgers wird die übertragene mechanische Spannung geringfügig. Die direkte Applizierung der Nuten in den Federkörper, ist jedoch insbesondere für gröBere Bauteile sehr aufwendig und sollte vermieden werden. 


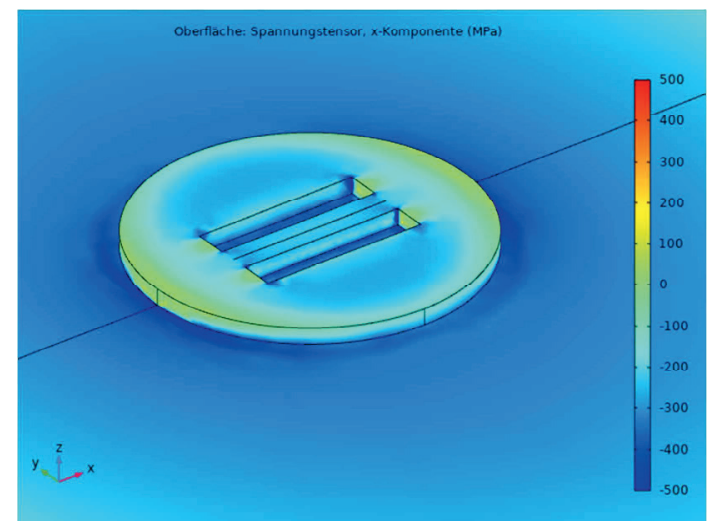

Abb. 6: FEM-Simulation der geschlitzten Montageträger, hohe mechanische Spannung in Richtung $x$, Belastung wie $A b b .4$

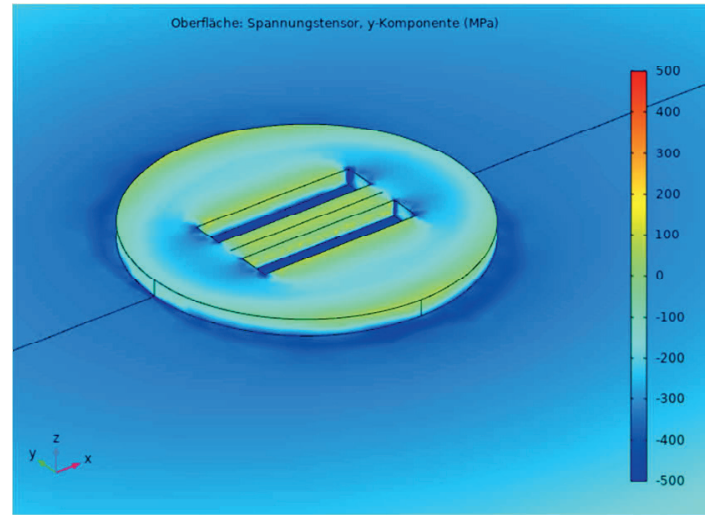

Abb. 7: FEM-Simulation der geschlitzten Montageträger, geringe mechanische Spannung in Richtung y

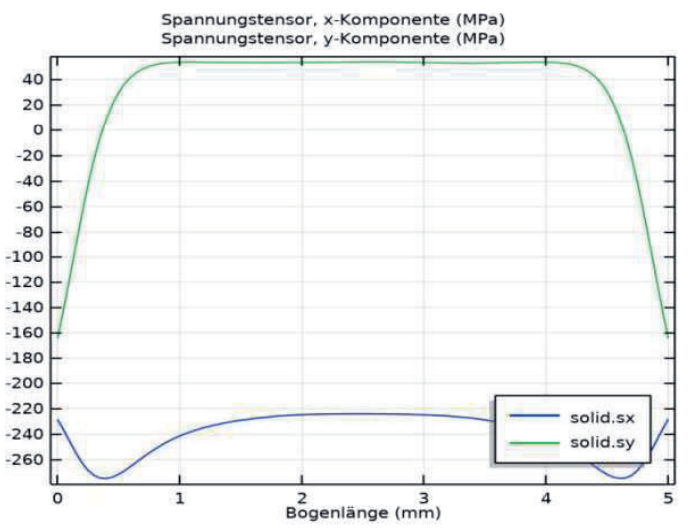

Abb. 8: FEM-Simulation der geschlitzten Montageträger, mechanische Spannung in Richtung $x$ und $y$

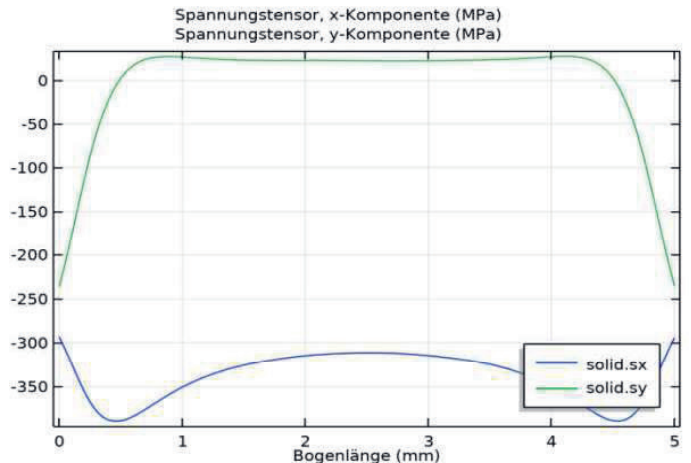

Abb. 9: FEM-Simulation, Nut direkt im Schraubenkopf, ohne Montageträger, mechanische Spannung in Richtung $x$ und $y$

\section{Beispielanwendung am Schraubenkopf}

Am Beispiel einer Schraube wird die Funktion gezeigt. Dazu werden Sechskantschrauben M16*1,5 verwendet. Der Steg, bzw. die Nuten wurden in den Schraubenkopf eingearbeitet. Dies erwies sich für die geringe Stückzahl zweckmäßiger. Die Abb. 10 zeigt die Struktur. Soll zusätzlich die Richtung der Belastung bestimmt werden können 3 Si-DMS um $120^{\circ}$ auf dem Kopf angeordnet werden, Abb. 10c. Die Si-DMS werden mit einer Glasfritte auf den Steg gefügt und mittels Drahtbondtechnik elektrisch kontaktiert.

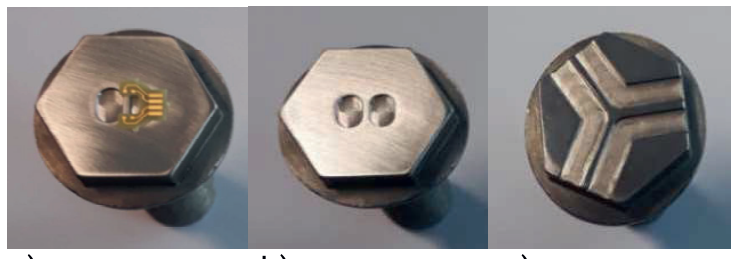

a)

b)

C)

Abb. 10: Schraubenkopf mit eingearbeiteten Steg

Die folgende Darstellung widmet sich der erreichten Ergebnisse. Es wurden zwei verschiedene Prüfvorrichtungen verwendet. Die in der Abb. 11 dargestellte Vorrichtung dient zum hydraulischen Verspannen der Schrauben. Durch die Druckzylinder wird die Schraube belastet, der Referenzsensor, ein Ringkraftsensor befindet sich unter dem Schraubenkopf. 


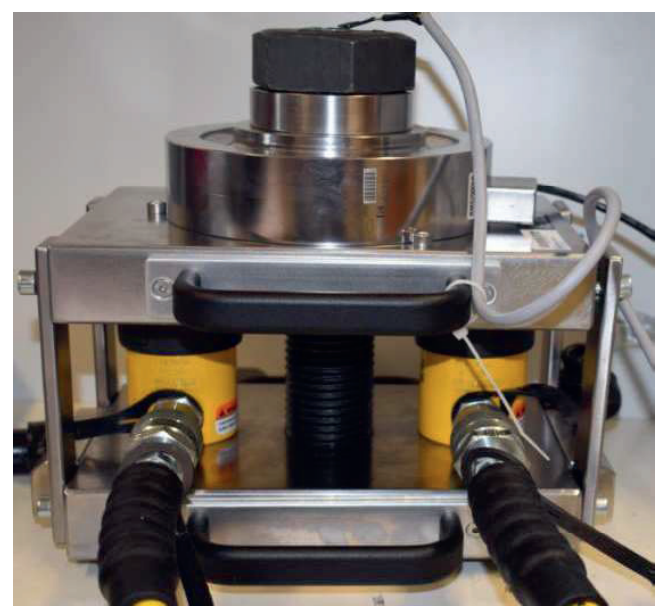

Abb. 11: Versuchsaufbau, hydraulisch verspannt, M48 8.8 als Beispiel

Zur Überprüfung des Einflusses des Anzugsmomentes wurde eine ähnliche Vorrichtung verwendet, die Vorspannkraft wurde mittels Drehmoment über die Schraubenmutter aufgebracht. Zur Darstellung der Wirksamkeit der Kompensation der Quereinflüsse ist in Abb. 12 das Ergebnis der Bestimmung der Vorspannkraft ohne Steg, planer Schraubenkopf, gezeigt. Die Messspanne ist durchaus ausreichend, resultiert aber aus Ungenauigkeiten in der Positionierung des Si-DMS. Die Schraube wurde 10 mal bis auf ca. $130 \mathrm{kN}$ belastet und vollständig entlastet. Die Kompensation der Quereinflüsse sollte einen Zuwachs der Messspannung um das 20 -fache ergeben.

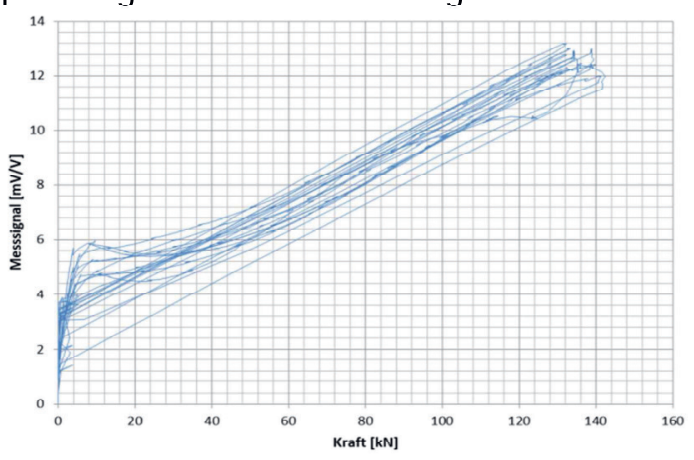

Abb. 12: Messung auf dem Schraubenkopf ohne Steg

In Abb. 13 sind die Untersuchungen der Schrauben mit der Kompensation dargestellt. Die Schraube wurde 10 mal be- und entlastet, sowohl hydraulisch als auch mittels Drehmoment.

Die Abb. 14 zeigt den Versuchsablauf. Sehr gut zu sehen ist aus beiden Abbildungen 13 und 14 die Unabhängigkeit des Messverfahrens von dem Anzugsverfahren. Eine wesentliche Fehlerquelle stellen die Referenzsensoren dar, es handelte sich um zwei verschiedene Ringkraftsensoren und die Wiederholgenauig- keit der Auflagepunkte der Schrauben auf dem Sensor konnte nicht überprüft werden.

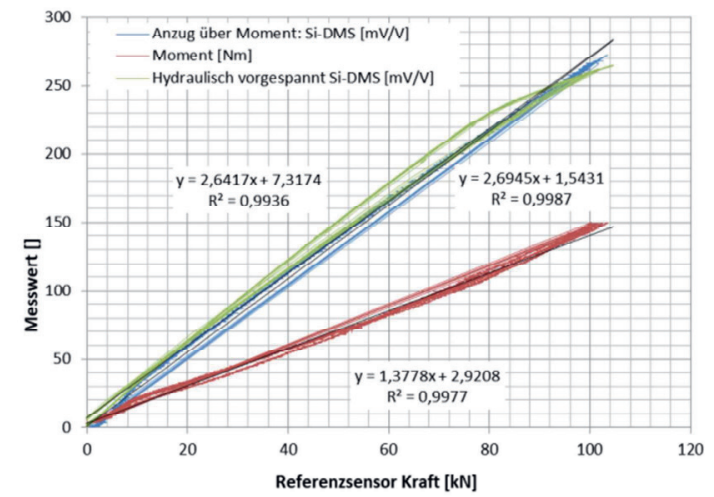

Abb. 13: Messwerte, Anzugsverfahren Drehmoment, hydraulisch verspannt über Referenzsensor (kN)

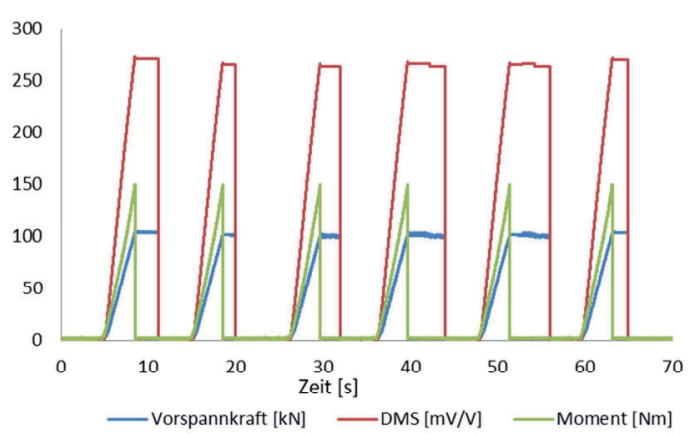

Abb. 14: Messwerte, Anzugsverfahren Drehmoment

Die nächste Frage stellt sich in Bezug auf die Kalibrierung. Wünschenswert ist eine Einpunktkalibration, das bedeutet, dass die Steigung in einem Toleranzbereich identisch ist, sich aber der Nullpunkt fertigungsbedingt variiert. Die Abb. 15 zeigt 10 Messgraden von je 3 verschiedenen Schrauben. Da bei diesem Anwendungsfall nur die oberen $20 \%$ des Messbereiches von Interesse sind ist die gezeigte Messgenauigkeit ausreichend. Weiter erhöht werden kann die Genauigkeit durch eine maschinelle Fertigung. Die wesentlichen Abweichungen betreffen die Position und die Dicke aller Materialien unter dem Si-DMS.

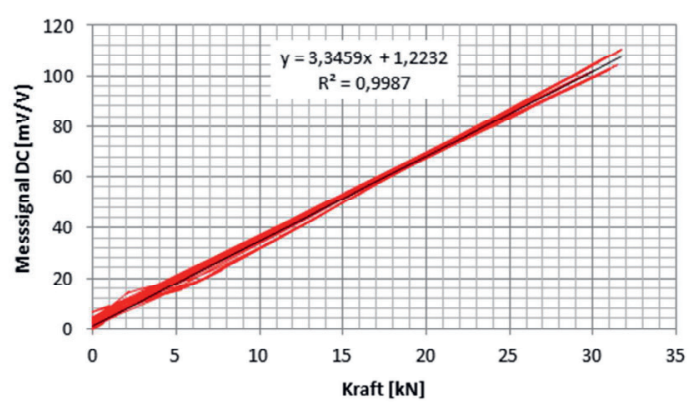

Abb. 15: Messgraden an 3 Schrauben 


\section{Beispielanwendung am Rohr}

Eine ähnliche Konstellation ergibt sich bei der Aufgabe den Druck in einem Rohr mittels eines Si-DMS zu bestimmen. Ein langgestrecktes Rohr weißt durch die wesentlich größere Länge im Verhältnis zum Durchmesser eine ausgeprägte Anisotropie der mechanischen Spannungen auf.

Die Abb. 16 verdeutlicht diesen Sachverhalt. Zur besseren Montage des Si-DMS und zur Erhöhung der Sensitivität wurde das Rohr abgeflacht. Das Rohr ist zusätzlich noch verstärkt um die Fläche einfacher zu gestallten. Die Abb. 17 zeigt die Verteilung der mechanischen Spannungen. Ausgewertet wird die Komponente in Richtung $x$.

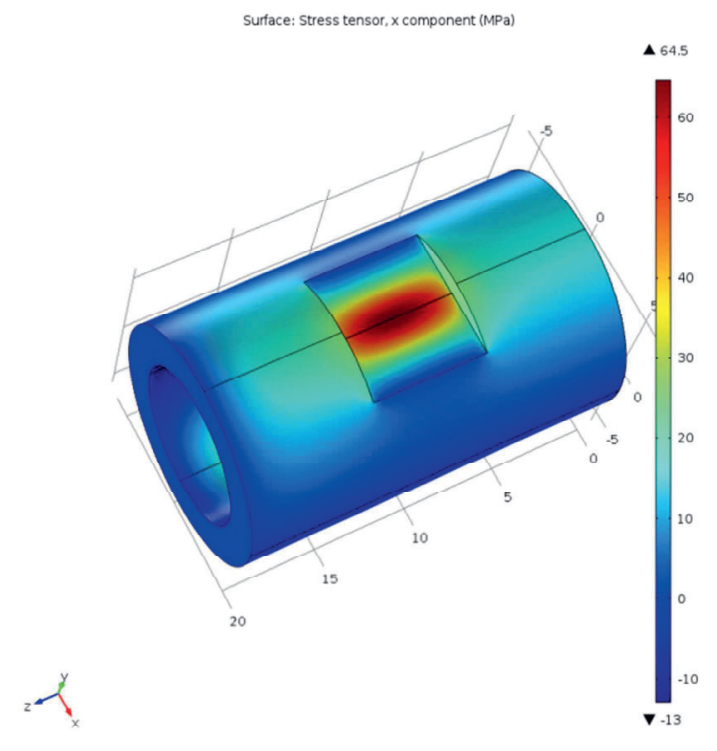

Abb. 16: Einfache Druckmessung im Rohr, Simulation 100 bar

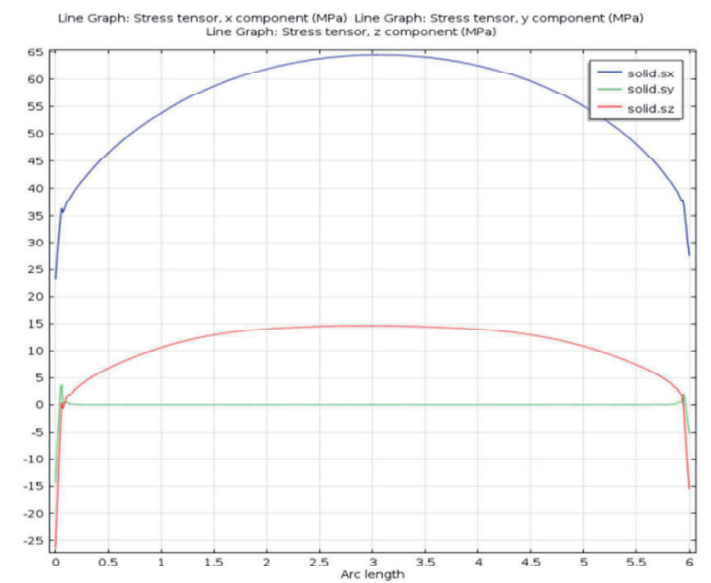

Abb. 17: Spannungsverteilung der einfachen Druckmessung im Rohr, Simulation $100 \mathrm{bar}$

\section{Fazit}

Es konnte gezeigt werden, dass mit der Anordnung von Sensoren auf dem Schraubenkopf die Vorspannkraft verlässlich bestimmt werden kann. Ein weitgehend kalibrierfreier Aufbau kann realisiert werden, wenn die Position der Sensoren auf dem Schraubenkopf exakt eingestellt werden. In diesem Fall wird es ausreichen nur den Nullpunkt zu kalibrieren, die Empfindlichkeit der untersuchten Sensoren lag in einer sehr engen Bandbreite. Die Langzeitstabilität konnte nur in Stunden untersucht werden, erforderlich sind aber Jahre, dies wird noch weitere Zeit benötigen.

\section{Literatur}

[1] J. Rausch, Entwicklung und Anwendung miniaturisierter piezoresistiver Dehnungsmesselemente, (2012)

[2] Th. Frank, M. Khatri, M. Fiedler, A. Gruen, M. Kermann, A. Cyriax, Th. Ortlepp, Miniaturized silicon strain gauge elements to accurately measure mechanical quantities, AMA Conferences 2015, Germany, (19.- 21.05.2015)

[3] Th. Frank, A. Grün, M. Kermann, A. Cyriax, A. Steinke, Th. Ortlepp, Diagnoseprüfung und Monitoring an Verbindungselementen mit Hilfe von Silizium-Dehnmessstreifen, 19. ITG/GMAFachtagung - Sensoren und Messsysteme 2018, Nürnberg, Deutschland, (26.-27.06.2018)

[4] Th. Frank, A. Grün, M. Kermann, A. Cyriax, A. Steinke, Th. Ortlepp, Monitoring von Schraubverbindungen Verbindungselementen mit Hilfe von Silizium-Dehnmessstreifen, Technische Sicherheit, Bd. 8, Nr. 9 (2018); 01.09.2018

\section{Danksagung}

Wir danken dem Bundesministerium für Wirtschaft und Energie (BMWi), dem Bundesministeriums für Bildung und Forschung (BMBF) und der Europäischen Kommission für die finanzielle Unterstützung der Projekte ACBrücke (MF150187), Bavi (49MF180012), HyPros-D (03ZZ0724D), Mikado (49MF18016 3), Screw Fixing Monitoring (ZF4001703HB8), SiStGAP (MF150091) und Smarter Si (ICT-022014). 